Also available at http://amc-journal.eu

ISSN 1855-3966 (printed edn.), ISSN 1855-3974 (electronic edn.)

ARS MATHEMATICA CONTEMPORANEA 10 (2016) 411-425

\title{
An infinite class of movable 5-configurations
}

\author{
Leah Wrenn Berman *
}

Department of Mathematics and Statistics, University of Alaska Fairbanks, Fairbanks, Alaska, USA

Elliott Jacksch

Seattle, Washington, USA

Lander Ver Hoef

NOAA Corps of Commissioned Officers, Ketchikan, Alaska, USA

Received 11 September 2015, accepted 3 March 2016, published online 13 April 2016

\begin{abstract}
A geometric 5-configuration is a collection of points and straight lines, typically in the Euclidean plane, in which every point has 5 lines passing through it and every line has 5 points lying on it; that is, it is an $\left(n_{5}\right)$ configuration for some number $n$ of points and lines. Using reduced Levi graphs and two elementary geometric lemmas, we develop a construction that produces infinitely many new 5-configurations which are movable; in particular, we produce infinitely many 5-configurations with one continuous degree of freedom, and we produce 5-configurations with $k-2$ continuous degrees of freedom for all odd $k>2$.
\end{abstract}

Keywords: Configurations, incidence geometry.

Math. Subj. Class.: 51A20, 51A45, 51E30, 05B30

A geometric $k$-configuration is a collection of points and straight lines, typically in the Euclidean plane, where every point lies on $k$ lines and every line passes through $k$ points. Geometric 3-configurations have been studied since the mid-1800s, and geometric 4-configurations since the late 1900s, with the first intelligible drawing of a 4-configuration appearing in a 1990 paper by Grünbaum and Rigby [15]. However, the situation for more highly incident configurations, that is, for $\left(p_{q}, n_{k}\right)$ configurations with at least one of $q$, $k \geq 4$, is poorly understood in general.

Two constructions that produce infinite families of 5-configurations with a reasonably small number of points and lines are known [7,9]. The $\left(48_{5}\right)$ configuration shown in Figure 1a

\footnotetext{
* Research supported by a grant from the Simons Foundation (\#209161 to L. Berman)

E-mail addresses: lwberman@alaska.edu (Leah Wrenn Berman), ecjacksch@alaska.edu (Elliott Jacksch), lander.verhoef@gmail.com (Lander Ver Hoef)
} 
is the smallest known geometric 5-configuration and is the smallest example of the construction in [9]; a reasonably small example of the construction discussed in [7] is shown in Figure 1b (the smallest example is not intelligible at small scale). In his monograph on configurations [14, Section 4.1], Grünbaum spends only 5 pages (mostly pictures) discussing the little that is known about 5-configurations.

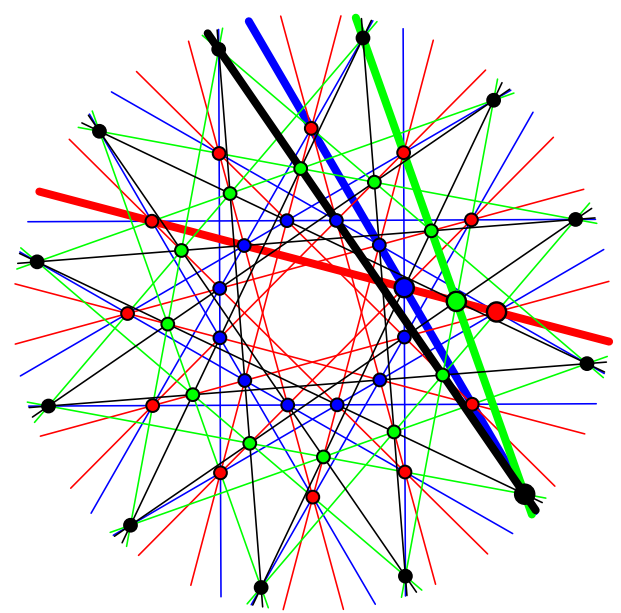

(a) A $(485)$ configuration with 4 symmetry classes of points and lines

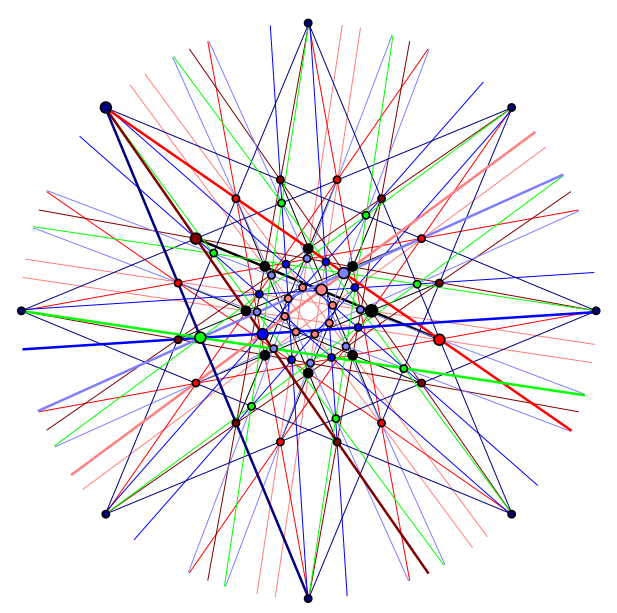

(b) A (645) configuration with 8 symmetry classes of points and lines

Figure 1: Examples of known small 5-configurations

In this paper, we present a new construction that produces infinitely many new geometric 5-configurations which are movable: that is, there is at least one continuous degree of freedom in the construction while fixing 4 points in general position. This construction significantly generalizes the construction presented in [9] and removes the need to complete the construction via a continuity argument, instead providing an entirely ruler-and-compass construction for those configurations, given an initial $m$-gon. The new construction technique uses two elementary geometric lemmas, the Circumcircle Construction Lemma and the Crossing Spans Lemma, which previously have been used separately in other configuration construction techniques.

\section{Definitions; Levi and reduced Levi graphs}

Given any $\left(p_{q}, n_{k}\right)$ configuration, whether geometrically realizable or not, it is possible to construct a corresponding bipartite graph, called a Levi graph, which has one white vertex for each point of the configuration and one black vertex for each line of the configuration, with two vertices in the graph incident if and only if the corresponding point and line are incident in the configuration. More details on Levi graphs and configurations may be found in Grünbaum [14, Section 1.4] and Coxeter [12].

We say that a geometric $k$-configuration is symmetric if there exist non-trivial isometries 
of the Euclidean plane that map the configuration to itself. Note that in other places in the literature, the word 'symmetric' has been used to mean $\left(p_{q}, n_{k}\right)$ configurations where $q=k$ (and thus $p=n$ ), i.e., $k$-configurations. Since we are interested in emphasizing the geometric nature of the configuration, we-following Grünbaum [14, p. 16]-refer to $k$-configurations as balanced, and reserve the word 'symmetric' to refer to the geometric structure. The symmetry class of an element (point or line) is the orbit of the element under the symmetry group of the configuration. If a geometric configuration has the property that every symmetry class under some fixed cyclic subgroup of the geometric symmetry group contains the same number of elements, then the configuration is called polycyclic; polycyclic configurations were first described by Boben and Pisanski [11].

Given a polycyclic geometric configuration with cyclic symmetry group $\mathbb{Z}_{m}$, it is possible to construct an edge-labelled bipartite graph, called the reduced Levi graph, by associating one vertex of the graph to each symmetry class of points and of lines in the configuration, and connecting two vertices of the graph with an edge precisely when the corresponding elements of the configuration are incident. Suppose the elements of each symmetry class of elements are labelled cyclically counterclockwise, beginning from some chosen 0th element in each class; for example, line class $L$ is labelled $(L)_{0}, \ldots,(L)_{m-1}$ and vertex class $v$ is labelled $(v)_{0}, \ldots,(v)_{m-1}$. If for each $i$, line $L_{i}$ and vertex $v_{i+a}$ are incident (with indices computed modulo $m$ ), the corresponding directed edge in the reduced Levi graph from vertex $L$ to vertex $v$ is labelled $a$; in the case where $L_{i}$ and vertex $v_{i}$ are incident (that is, where $a=0$ ), then we use an undirected thick edge. When vertices $v_{i}$ and $v_{i+a}$ both lie on line $L_{i}$, or from an alternate point of view, when lines $L_{i}$ and $L_{i-a}$ intersect at point $v_{i}$,

then the reduced Levi graph contains a double arc

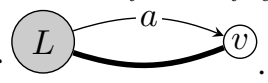

If $p$ and $q$ are any two points, we denote the line $L$ passing through $p$ and $q$ as $p \vee q$. Similarly, if $L$ and $M$ are any two lines, we denote their point of intersection as $L \wedge M$ (possibly at infinity if $L \| M)$. Given points $v_{0}, \ldots, v_{m-1}$ that form the vertices of a regular $m$-gon centered at $\mathcal{O}$, we say that a line is span $b$ if it passes through $v_{i}$ and $v_{i+b}$ for some $i$, with

all indices computed modulo $m$; span $b$ lines correspond to double arcs

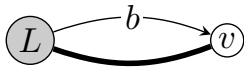
in the reduced Levi graph. A circle $C$ is a circumcircle of span $b$ if it passes through $v_{i}, \mathcal{O}$, and $v_{i-b}$ for some $i$; to specify which $i$, we say that $C$ is a circumcircle of span $b$ through $v_{d}$. (Note that span $b$ lines are constructed by moving counterclockwise from the initial point, and span $b$ circumcircles by moving clockwise!)

\section{Two construction lemmas}

In 2006, one of the authors (LWB) discovered the Crossing Spans Lemma [3] (somewhat restated here):

Lemma 2.1 (Crossing Spans Lemma (CSL)). Given a regular $m$-gon with vertices cyclically labelled as $u_{0}, u_{1}, \ldots, u_{m-1}$ and lines $L_{i}=u_{i} \vee u_{i+a}$ of span a and $M_{i}=u_{i} \vee u_{i+b}$ of span $b$, where $1 \leq a \neq b<\frac{m}{2}$, suppose that $v_{0}$ is an arbitrary point on $M_{0}$ (different from $u_{0}, u_{b}$ to avoid degeneracies), and construct other points $v_{i}$ to be the rotations of $v_{0}$ through $\frac{2 \pi i}{m}$. Let $N_{i}=v_{i} \vee v_{i+a}$ and let $w_{i}=N_{i} \wedge N_{i-b}$. Then $w_{i}$ also lies on $L_{i}$.

Although easy to state and prove, the Crossing Spans Lemma has been used to produce a 


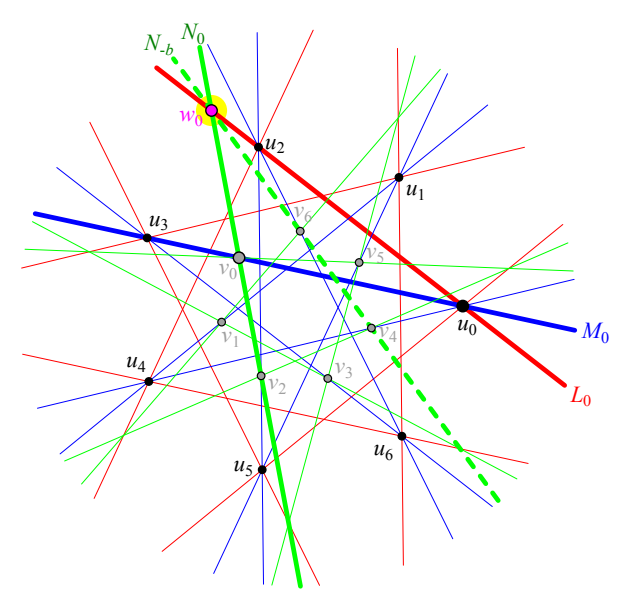

(a) Illustrating the Crossing Spans Lemma; $m=$ $7, a=2, b=3$. Only point $w_{0}$ in class $w$ has been shown, to better illustrate that the three lines $L_{i}, N_{i-b}$, and $N_{i}$ really do intersect three at a time (that is, no almost-incidences are covered by points).

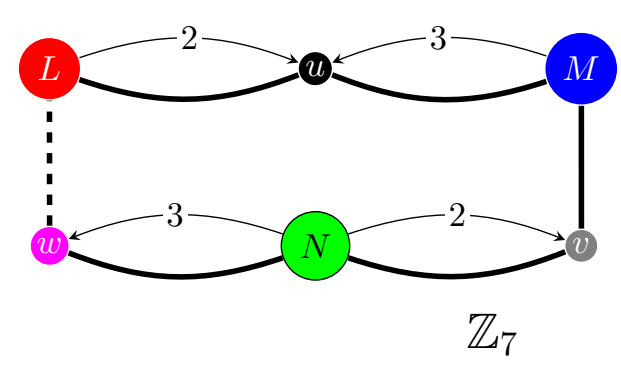

(b) The reduced Levi graph corresponding to Figure 2; the dashed edge corresponds to the forced incidence.

Figure 2: Illustrating the Crossing Spans Lemma

number of novel constructions for configurations [3, 5, 8, 9]. The Crossing Spans Lemma and its associated reduced Levi graph "gadget" are shown in Figure 2.

In fact, it is straightforward to show (by relabelling symmetry classes and applying duality arguments) that given either of the labelled subgraphs in a reduced Levi graph that are shown in Figure 3, the incidence given by the dashed line is induced, where white nodes correspond to point classes and gray nodes to line classes. These subgraphs, with various choices of labels, are used extensively in the proof of Theorem 4.1.
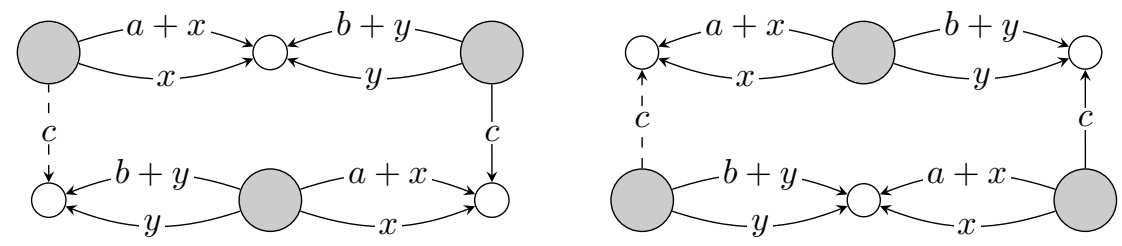

Figure 3: In either of these subgraphs in a reduced Levi graph (over $\mathbb{Z}_{m}$ ), the dashed line corresponds to a forced incidence via the CSL; , $c, x, y$ are integers between 0 and $m-1$, and $1 \leq a \neq b<\frac{m}{2}$. Gray vertices correspond to line classes and white vertices to point classes. In the construction in Section 4, we typically take $c=0, x=0$, and $y=0$ or $\delta$.

In [14, p. 116-118], Branko Grünbaum described a geometric technique to constructing a certain class of 3-configurations. This technique was extended in [7] to the Circumcircle Construction Lemma. Although the lemma can be stated as a more general incidence theorem [8], we state it as follows in order to facilitate the main construction in Section 4. 
Lemma 2.2 (Circumcircle Construction Lemma (CCL)). Let $v_{0}, v_{1}, \ldots, v_{m-1}$ and $w_{0}, w_{1}, \ldots, w_{m-1}$ form the vertices, labelled cyclically counterclockwise, of two regular convex $m$-gons centered at $\mathcal{O}$. The point $w_{0}$ lies on the circle passing through $v_{d}, v_{d-b}, \mathcal{O}$ if and only if the points $w_{0}, w_{b}, v_{d}$ are collinear.

That is, if $w_{0}$ lies on the circumcircle of span $b$ through $v_{d}$, then the line $L_{0}$ of span $b$ through $w_{0}$ passes through $v_{d}$, and conversely. By symmetry, the line $L_{-d}$ will also pass through the point $w_{0}$, and in general, if $w_{0}$ is defined to also lie on some other line $M_{0}$, then each rotated image $w_{i}$ will lie on the three lines $L_{i}, L_{i-d}$ and $M_{i}$. The Circumcircle Construction Lemma, along with its reduced Levi graph structure, is illustrated in Figure 4.

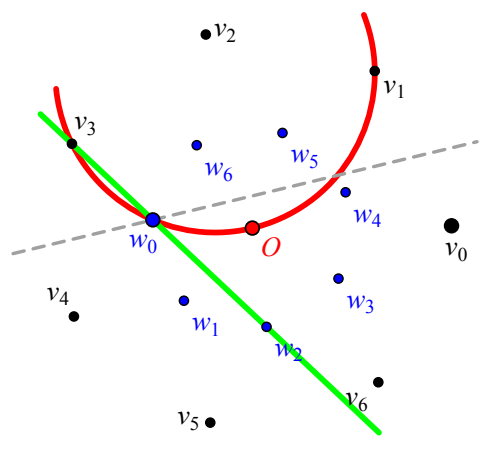

(a) Illustrating the Circumcircle Construction Lemma; $m=7, b=2, d=3$. The green line is $L_{0}$, and the dashed gray line is a possible other line $M_{0}$ passing through $w_{0}$ (i.e., $w_{0}$ could be defined as the intersection of $M_{0}$ and $C$ ); other elements of line classes $L$ and $M$ have been suppressed for clarity.

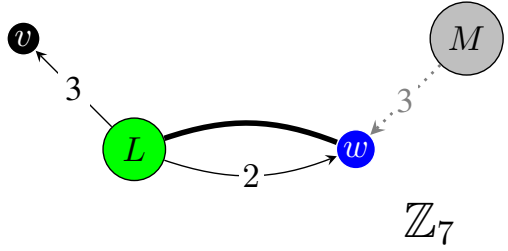

(b) The "gadget" in a reduced Levi graph corresponding to Figure $4 \mathrm{a}$. (The connection between $w$ and $M$ is optional, depending on whether there happens to be a line $M_{0}$ passing through $w_{0}$; this is the typical situation in applications of the CCL.)

Figure 4: The Circumcircle Construction Lemma.

\section{Celestial 4-configurations}

The building blocks for the new construction of 5-configurations presented in Section 4 are the celestial 4-configurations, which are configurations that have the property that every point has two lines from each of two symmetry classes of lines passing through it, and every line has two points from each of two symmetry classes of points lying on it. An example of such a configuration is shown in Figure 5, along with a general reduced Levi graph. Celestial 4-configurations were first described in detail (aside from a handful of examples, e.g., [15, 16]) in Boben and Pisanski's article Polycyclic Configurations [11], as the main class of 4-configurations analyzed in that paper. Their description was expanded in Grünbaum's monograph Configurations of Points and Lines [14, Sections 3.5-3.8], although in that chapter, he unfortunately called them $k$-astral configurations (even though as he defined previously [14, p. 34], a $k$-astral configuration is simply a configuration with $k$ symmetry classes of points and of lines, and there exist $k$-astral 4-configurations that are not $k$-celestial [13]). 
Every $k$-celestial 4-configuration can be described by a celestial symbol

$$
m \#\left(s_{1}, t_{1} ; \ldots ; s_{k}, t_{k}\right)
$$

that satisfies four axioms:

Axiom 1: (order condition) $s_{i} \neq t_{i} \neq s_{i+1}$ (with indices taken modulo $m$ )

Axiom 2: (even condition) $\sum_{i=1}^{k}\left(s_{i}-t_{i}\right)=2 \delta$ for some integer $\delta$

Axiom 3: (cosine condition) $\prod_{i=1}^{k} \cos \left(\frac{s_{i} \pi}{m}\right)=\prod_{i=1}^{k} \cos \left(\frac{t_{i} \pi}{m}\right)$

Axiom 4: (substring condition) no substring $s_{i}, t_{i} ; \ldots ; s_{j}, t_{j}$ or $t_{i} ; s_{i+1}, \ldots, t_{j} ; s_{j+1}$ satisfies the previous axioms.

A symbol satisfying the 4 axioms is said to be valid. Although celestial 4-configurations are probably the most well-understood class of 4-configuration, they are still poorly understood in general. The collection of 2-celestial configurations is completely classified ([2], with a clearer proof in [14, p. 210-211]), but general $k$-celestial configurations are not completely classified, and the problem appears to be non-tractable (since it depends on being able to solve certain trigonometric diophantine equations). However, some known families of valid $k$-celestial configurations, primarily for $k=3,4$, were presented in [1].

Given a valid symbol, there is a corresponding cohort $m \# S ; T$, where $S=\left\{s_{1}, \ldots, s_{k}\right\}$ and $T=\left\{t_{1}, \ldots, t_{k}\right\}$ (as sets), which corresponds to a collection of valid symbols; in particular, the sets in a cohort must satisfy the even and cosine conditions, and it must be possible to find an ordering of the $s_{i}$ and $t_{i}$ that satisfies the order condition.

To construct a $k$-celestial 4-configuration $m \#\left(s_{1}, t_{1} ; \ldots ; s_{k}, t_{k}\right)$ with $k$ point classes $v_{1}, \ldots, v_{k}$ and $k$ line classes $L_{1}, \ldots, L_{k}$, do the following:

Algorithm 1 (Constructing a celestial 4-configuration).

Input: A valid celestial symbol $m \#\left(s_{1}, t_{1} ; \ldots ; s_{k}, t_{k}\right)$.

1. Construct the vertices of a regular $m$-gon centered at $\mathcal{O}$, labelled $\left(v_{1}\right)_{0}, \ldots\left(v_{1}\right)_{m-1}$.

2. Let $L_{1}$ be the collection of lines of span $s_{1}$ with respect to point class $v_{1}$ : that is, let $\left(L_{1}\right)_{i}=\left(v_{1}\right)_{i} \vee\left(v_{1}\right)_{i+s_{1}}$.

3. Construct point class $v_{2}$ to be the set of $t_{1}$-st intersection points of the lines $L_{1}$ : that is, $\left(v_{2}\right)_{i}=\left(L_{1}\right)_{i} \wedge\left(L_{1}\right)_{i-t_{1}}$.

4. Continue in this fashion; line class $L_{2}$ is the set of lines of span $s_{2}$ with respect to point class $v_{2}$, point class $v_{3}$ is the set of $t_{2}$-nd intersection points of the lines $L_{2}$, etc., stopping after the construction of line class $L_{k}$.

Because the symbol $m \#\left(s_{1}, t_{1} ; \ldots ; s_{k}, t_{k}\right)$ is valid, the point class $v_{k+1}$ corresponds, as a set, to point class $v_{1}$, and in particular, $\left(v_{k+1}\right)_{0}=\left(v_{1}\right)_{\delta}$, where $2 \delta=\sum_{i=1}^{k}\left(s_{i}-t_{i}\right)$.

The general reduced Levi graph for the configuration $m \#\left(s_{1}, t_{1} ; \ldots ; s_{k}, t_{k}\right)$ is shown in Figure $5 \mathrm{~b} ; \delta$, the "twist" [11], is guaranteed to be an integer by the even condition. In 
general, the underlying graph for every reduced Levi graph of a celestial 4-configuration is a double cycle of even length; that is, an even cycle in which every edge is replaced by a pair of parallel edges.

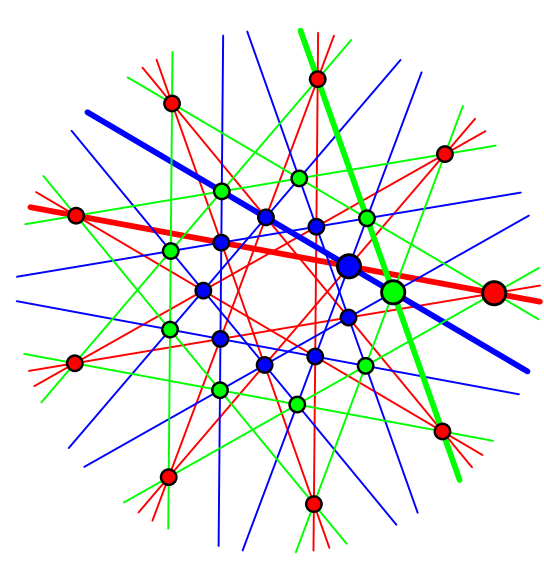

(a) The celestial 4-configuration 9\# 4,$3 ; 2,3 ; 1,3)$. The 0th element of each symmetry class is shown larger (points) or thicker (lines), and elements in different symmetry classes are distinguished by color (class 1 is red, class 2 is blue, and class 3 is green).

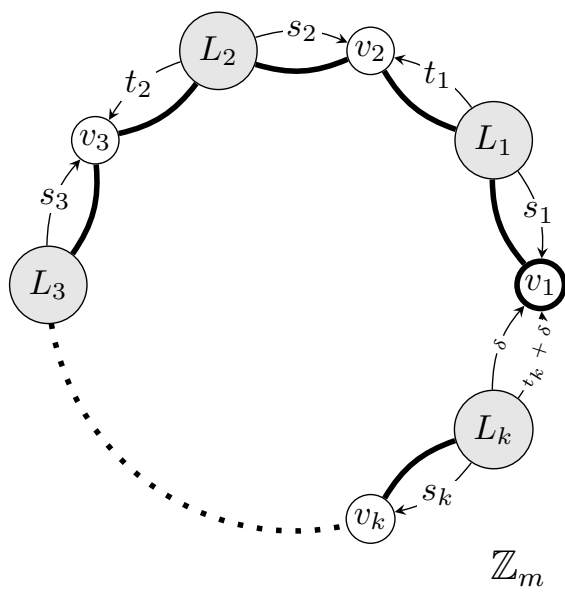

(b) The reduced Levi graph, a double cycle, for a general celestial 4-configuration, where $\delta=$ $\frac{1}{2} \sum_{i=1}^{k}\left(s_{i}-t_{i}\right)$.

Figure 5: Celestial 4-configurations

\section{Constructing movable 5-configurations}

The general idea of the construction is to produce a 5-configuration whose reduced Levi graph consists of concentric double cycles, each of which corresponds to a particular celestial 4-configuration, where the double cycles are successively linked by single edges by applying the CSL, and finally, the innermost cycle is linked to the outermost cycle using the CCL; if $k>2$ the construction will produce a movable 5-configuration. The reduced Levi graph is shown in Figure 6.

More specifically, the reduced Levi graph contains $k$ concentric double cycles, each of which corresponds to a $k$-celestial 4-configuration with cohort $m \# S ; T$ where $S \cap T=\varnothing$. If the outermost cycle corresponds to the configuration with symbol

$$
m \#\left(s_{1}, t_{1} ; s_{2}, t_{2} ; \ldots ; s_{k-1}, t_{k-1} ; s_{k}, t_{k}\right),
$$

then each successive cycle has the $s_{i}$ 's permuted cyclically one step while the $t_{i}$ 's remain fixed: that is, the second cycle has symbol

$$
m \#\left(s_{2}, t_{1} ; s_{3}, t_{2} ; \ldots ; s_{k}, t_{k-1} ; s_{1}, t_{k}\right),
$$

the third has symbol

$$
m \#\left(s_{3}, t_{1} ; s_{4}, t_{2} ; \ldots ; s_{1}, t_{k-1} ; s_{2}, t_{k}\right)
$$


and so on, so that the innermost cycle has symbol

$$
m \#\left(s_{k}, t_{1} ; s_{1}, t_{2} ; \ldots ; s_{k-2}, t_{k-1} ; s_{k-1}, t_{k}\right) \text {. }
$$

The point classes of the celestial configuration corresponding to cycle $j$ are labelled $v_{1}^{j}, \ldots$ $v_{k}^{j}$ and the line classes $L_{1}^{j}, \ldots L_{k}^{j}$; that is, the superscript indicates the cycle, and the subscript the symmetry class in the celestial configuration. In Figure 6, the first point class of each celestial configuration is highlighted.

Given a valid configuration symbol $m \#\left(s_{1}, t_{1} ; \ldots ; s_{k}, t_{k}\right)$ with cohort $m \# S ; T$ with the property that $S \cap T=\varnothing$, the geometric construction algorithm to produce a 5-configuration with $k-2$ continuous degrees of freedom is given in Algorithm 2. If $k=2$ the configuration is static and has been described previously in [9]; however, the construction algorithm given here, which uses the CCL to complete the construction, eliminates the need for completing the configuration via a continuity argument as described in that paper.

Algorithm 2 (Constructing a 5-configuration).

Input: A valid celestial symbol $m \#\left(s_{1}, t_{1} ; \ldots ; s_{k}, t_{k}\right)$ with the property that $S \cap T=\varnothing$.

1. Construct the first $k$-celestial 4-configuration with symbol $m \#\left(s_{1}, t_{1} ; \ldots ; s_{k}, t_{k}\right)$, with point classes $v_{1}^{1}, \ldots, v_{k}^{1}$ and line classes $L_{1}^{1}, \ldots, L_{k}^{1}$.

2. If $k>2$, for $j=2, \ldots, k-1$ :

(a) Place a new point $\left(v_{1}^{j}\right)_{0}$ arbitrarily on line $\left(L_{1}^{j-1}\right)_{0}$, and construct the rest of the points $\left(v_{1}^{j}\right)_{i}$ in point class $v_{1}^{2}$ by rotating $\left(v_{1}^{1}\right)_{0}$ by $\frac{2 \pi i}{m}$ for $i=0, \ldots m-1$.

(b) Using the point class $v_{1}^{j}$ as the starting $m$-gon, construct the configuration

$$
m \#\left(s_{j}, t_{1} ; s_{j+1}, t_{2} ; \ldots ; s_{j-2}, t_{k-1} ; s_{j-1}, t_{k}\right)
$$

(where the sequence $s_{1}, s_{2}, \ldots, s_{k-1}, s_{k}$ has been cyclically permuted $j$ steps but the sequence $t_{1}, \ldots, t_{k}$ remains fixed).

3. To construct the $k$-th celestial configuration:

(a) Construct a circumcircle $\mathcal{C}$ of span $s_{k}$ through $\left(v_{1}^{1}\right)_{c}$, choosing $c$ (and varying continuous parameters if possible/necessary) so that $\mathcal{C}$ intersects line $\left(L_{1}^{k-1}\right)_{0}$.

(b) Let $\left(v_{1}^{k}\right)_{0}$ be the intersection of $\mathcal{C}$ with line $\left(L_{1}^{k-1}\right)_{0}$, and let $\left(v_{1}^{k}\right)_{i}$ be the rotation of $\left(v_{1}^{k}\right)_{0}$ through $\frac{2 \pi i}{m}$ about $\mathcal{O}$.

(c) Construct configuration

$$
m \#\left(s_{k}, t_{1} ; s_{1}, t_{2} ; \ldots ; s_{k-2}, t_{k-1} ; s_{k-1}, t_{k}\right)
$$

using the points $\left(v_{1}^{k}\right)_{i}$ as the initial set of points.

Theorem 4.1. Algorithm 2 , beginning with $m \#\left(s_{1}, t_{1} ; \ldots ; s_{k}, t_{k}\right)$, creates a valid 5 -configuration with $m k^{2}$ points, $m k^{2}$ lines and $k-2$ continuous degrees of freedom. 


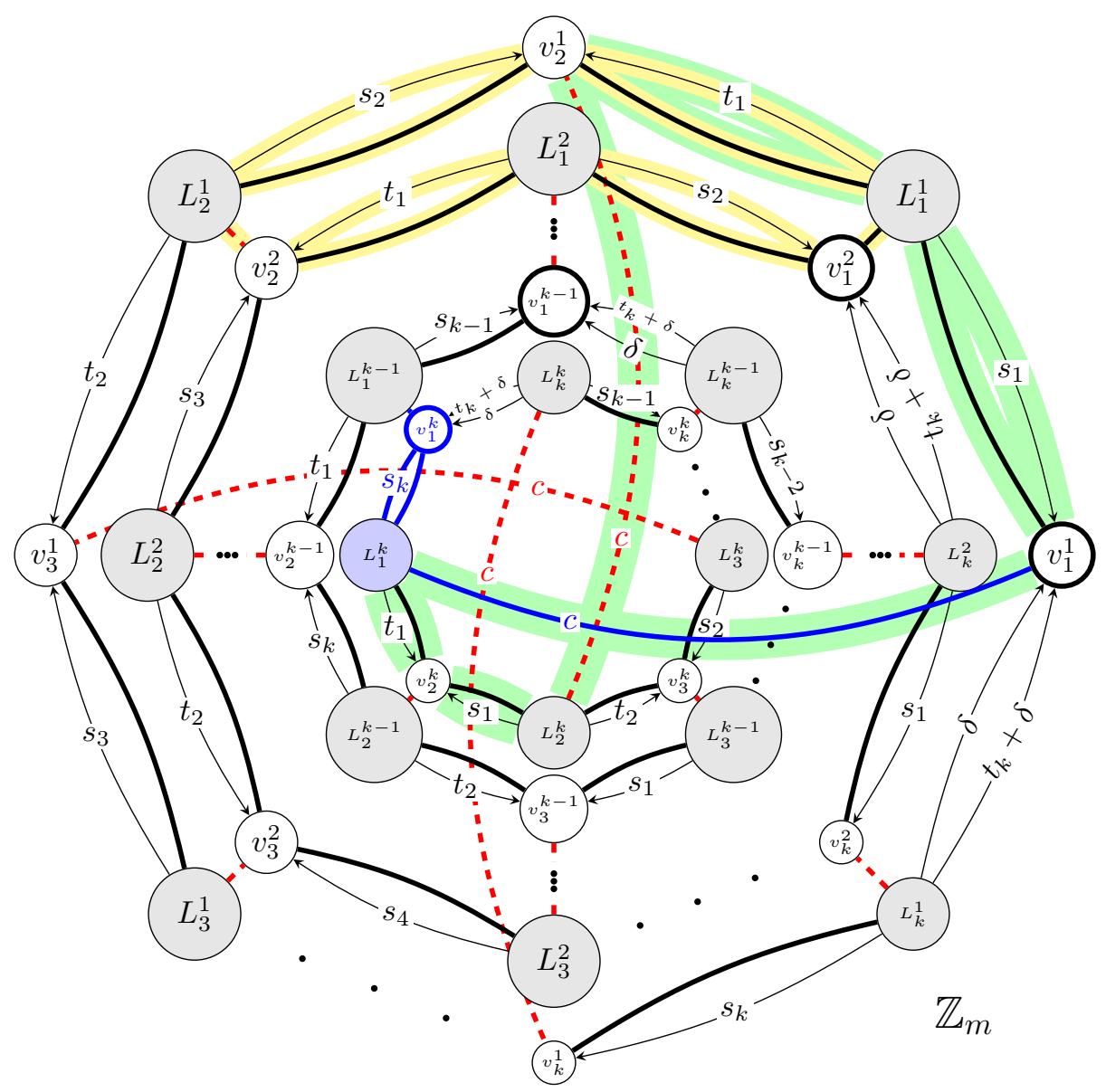

Figure 6: The reduced Levi graph, over $\mathbb{Z}_{m}$, for a movable 5-configuration with $k^{2}$ point classes and $k^{2}$ line classes. It consists of $k$ concentric double cycles, each corresponding to a particular celestial 4-configuration, with the double cycles linked by arcs. The arcs shown red and dashed are induced by the Crossing Spans Lemma, with example CSL gadgets inducing the dashed edges highlighted in yellow and green, while the structure shown in blue is constructed via the Circumcircle Construction Lemma. 
Proof. First, note that Algorithm 2 constructs $k$ celestial configurations; each celestial confguration contains $k$ symmetry classes of points and of lines, and each symmetry class contains $m$ elements, for a total of $m k^{2}$ points and $m k^{2}$ lines.

Second, for $j=2, \ldots, k-1$, the point $\left(v_{1}^{j}\right)_{0}$ is placed arbitrarily on line $\left(L_{1}^{j-1}\right)_{0}$, for $(k-1)-2+1=k-2$ continuous degrees of freedom.

Thus, the nontrivial part of the proof is to show that every point lies on 5 lines, and every line passes through 5 points.

Recall that the symbol for celestial configuration $j$ is

$$
m \#\left(s_{j}, t_{1} ; s_{j+1}, t_{2} ; \ldots ; s_{j+\ell}, t_{\ell} ; \ldots ; s_{j-1}, t_{k}\right) .
$$

By construction, for each $j=1, \ldots, k-1$, each line $\left(L_{1}^{j}\right)_{i}$ passes through the point $\left(v_{1}^{j+1}\right)_{i}$ (that is, the first symmetry class of points in celestial configuration $j+1$ lies on the first symmetry class of lines in celestial configuration $j$ ), as well as through points $\left(v_{1}^{j}\right)_{i}$, $\left(v_{1}^{j}\right)_{i+s_{j}},\left(v_{2}^{j}\right)_{i}$, and $\left(v_{2}^{j}\right)_{i+t_{1}}$ from celestial configuration $j$.

By careful choice of labels and the Crossing Spans Lemma, it follows that for all $\ell=$ $2, \ldots, k-1$ (with $\ell$ indexing the symmetry classes in the celestial configuration $j$ ), each line $\left(L_{\ell}^{j}\right)_{i}$ passes through point $\left(v_{\ell}^{j+1}\right)_{i}$, as well as through points $\left(v_{\ell}^{j}\right)_{i},\left(v_{\ell}^{j}\right)_{i+s_{j+\ell}},\left(v_{\ell+1}^{j}\right)_{i}$ and $\left(v_{\ell+1}^{j}\right)_{i+t_{\ell}}$ from celestial configuration $j$.

A CSL gadget showing that points $v_{2}^{2}$ are incident with lines $L_{2}^{1}$ (dashed red line) beginning with the input that points $v_{1}^{2}$ are constructed incident with lines $L_{2}^{1}$ (solid black line) is highlighted in Figure 6 in yellow.

Finally, again by the CSL, line $\left(L_{k}^{j}\right)_{i}$ passes through point $\left(v_{k}^{j+1}\right)_{i}$, as well as through points $\left(v_{k}^{j}\right)_{i},\left(v_{k}^{j}\right)_{i+s_{j-1}},\left(v_{1}^{j}\right)_{i+\delta}$ and $\left(v_{1}^{j}\right)_{i+\delta+t_{k}}$ from the completion of the celestial configuration $j$.

Thus, for $j=1, \ldots, k-1$ (indexing the celestial configuration), $\ell=1, \ldots k$ (indexing the symmetry class in the celestial configuration) and $i=0, \ldots m-1$ (indexing the elements of the symmetry class) each line $\left(L_{\ell}^{j}\right)_{i}$ has 5 points lying on it. By inspection of the previous incidences, for $j=2, \ldots, k-1$, each point $\left(v_{\ell}^{j}\right)_{i}$ has 5 lines passing through it; however, points $\left(v_{\ell}^{1}\right)_{i}$ only have 4 lines passing through them so far.

However, in step 3 , we constructed $\left(v_{1}^{k}\right)_{0}$ be the intersection of $\mathcal{C}$ with line $\left(L_{1}^{k-1}\right)_{0}$, where $\mathcal{C}$ is a circle of span $s_{k}$ through $\left(v_{1}^{1}\right)_{c}$. By the Circumcircle Construction Lemma it follows that points $\left(v_{1}^{k}\right)_{0},\left(v_{1}^{k}\right)_{s_{k}}$ and $\left(v_{1}^{1}\right)_{c}$ are collinear; that is line $\left(L_{1}^{k}\right)_{0}$, which is span $s_{k}$ with respect to the points $v_{1}^{k}$ by construction, passes through point $\left(v_{1}^{1}\right)_{c}$. By symmetry, it follows that line $\left(L_{1}^{k}\right)_{i}$ passes through $\left(v_{1}^{1}\right)_{i+c}$ for $i=0, \ldots, m-1$. (This is represented by the thick blue line connecting the inner and outer rings in Figure 6.) By construction of the $k$ th celestial configuration, it follows that line $\left(L_{1}^{k}\right)_{i}$ also passes through points $\left(v_{1}^{k}\right)_{i}$, $\left(v_{1}^{k}\right)_{i+s_{k}},\left(v_{2}^{k}\right)_{i}$ and $\left(v_{2}^{k}\right)_{i+t_{k}}$.

A final application of the Crossing Spans Lemma on gadgets connecting the inner and outer ring shows that symmetry class $L_{\ell}^{k}$ in the $k$-th celestial configuration is incident with symmetry class $v_{\ell}^{1}$ in the first celestial configuration. The CSL gadget showing that $L_{2}^{k}$ is incident with $v_{2}^{1}$ (dashed red curve), beginning with the fact that $L_{1}^{k}$ is incident with $v_{1}^{1}$ 
(thick blue curve) is highlighted in green in Figure 6. Specifically, for $\ell=2, \ldots k-1,\left(L_{\ell}^{k}\right)_{i}$ passes through $\left(v_{\ell}^{1}\right)_{i},\left(v_{\ell}^{k}\right)_{i},\left(v_{\ell}^{k}\right)_{i+s_{\ell-1}},\left(v_{\ell}^{k}\right)_{i},\left(v_{\ell}^{k}\right)_{i+t_{\ell}}$. Finally, $\left(L_{k}^{k}\right)_{i}$ passes through $\left(v_{k}^{1}\right)_{i},\left(v_{k}^{k}\right)_{i},\left(v_{k}^{k}\right)_{i+s_{k-1}},\left(v_{1}^{k}\right)_{i+\delta}$, and $\left(v_{1}^{k}\right)_{i+\delta+t_{k}}$. Thus, every point lies on 5 lines, and every line passes through 5 points.

\section{Some valid inputs for Algorithm 2}

Proposition 5.1. The smallest movable 5-configuration produced by Algorithm 2 uses $9 \#(4,3 ; 2,3 ; 1,3)$ (or another configuration with the same cohort) as its input and has 81 points and lines.

Proof. If $k=2$, Algorithm 2 produces static configurations. Inspection of a list of all valid symbols for small 3-celestial configurations (e.g., from [14, Table 3.7.1] or from the personal list of one of the the authors (LWB)) shows that the cohort $9 \#\{4,2,1\} ;\{3,3,3\}$ is the smallest cohort with disjoint sets.

This configuration is shown in Figure 7.

Theorem 5.2. There exist infinitely many 5-configurations with one continuous degree of freedom.

Proof. From [1] we know that

$$
2 q \#\{q-p, p, q-2 r\} ;\{q-r, r, q-2 p\}, \text { for } q \geq 4 \text { and } 0<p, r<q
$$

is a valid family of celestial 4-configuration cohorts.

Suppose that $r \neq p, r \neq \frac{q}{3}, p \neq \frac{q}{3}$ and $p+r \neq q$. Under these conditions, the sets $S$ and $T$ will always be disjoint. To see this, first note that $q-p \neq q-r$, because $p \neq r ; q-p \neq r$, because $p+r \neq q$; and $q-p \neq q-2 p$ because $p \neq 0$. Next, $p \neq q-r$ because $p+r \neq q$; $p \neq r$ by hypothesis; and $p \neq q-2 p$ since $p \neq q / 3$. Finally, $q-2 r \neq q-r$ because $r \neq 0 ; q-2 r \neq r$ since $r \neq q / 3$; and $q-2 r \neq q-2 p$ because $r \neq p$. Thus, the sets are disjoint. Hence the cohort is valid as input for Algorithm 2.

In particular, $p=1$ and $r=2$ produces the valid input cohort $2 q \#\{q-1,1, q-4\} ;\{q-$ $2,2, q-2\}$ for any $q \geq 4$.

Lemma 5.3. The cohort $3 q \#\left\{1,2, \ldots, 2^{k-1}\right\} ;\{\underbrace{q, q, \ldots, q}_{k}\}$ for $q=\frac{2^{k}+1}{3}, k$ odd and $k>2$ is a valid celestial cohort.

Proof. Note that the cohort $9 \#\{1,2,4\} ;\{3,3,3\}$ can be viewed as the case $k=3$ of this cohort.

To show the cohort is valid, we need to show that $q=\frac{2^{k}+1}{3}$ is an integer and that the cohort satisfies the cosine and even conditions. 


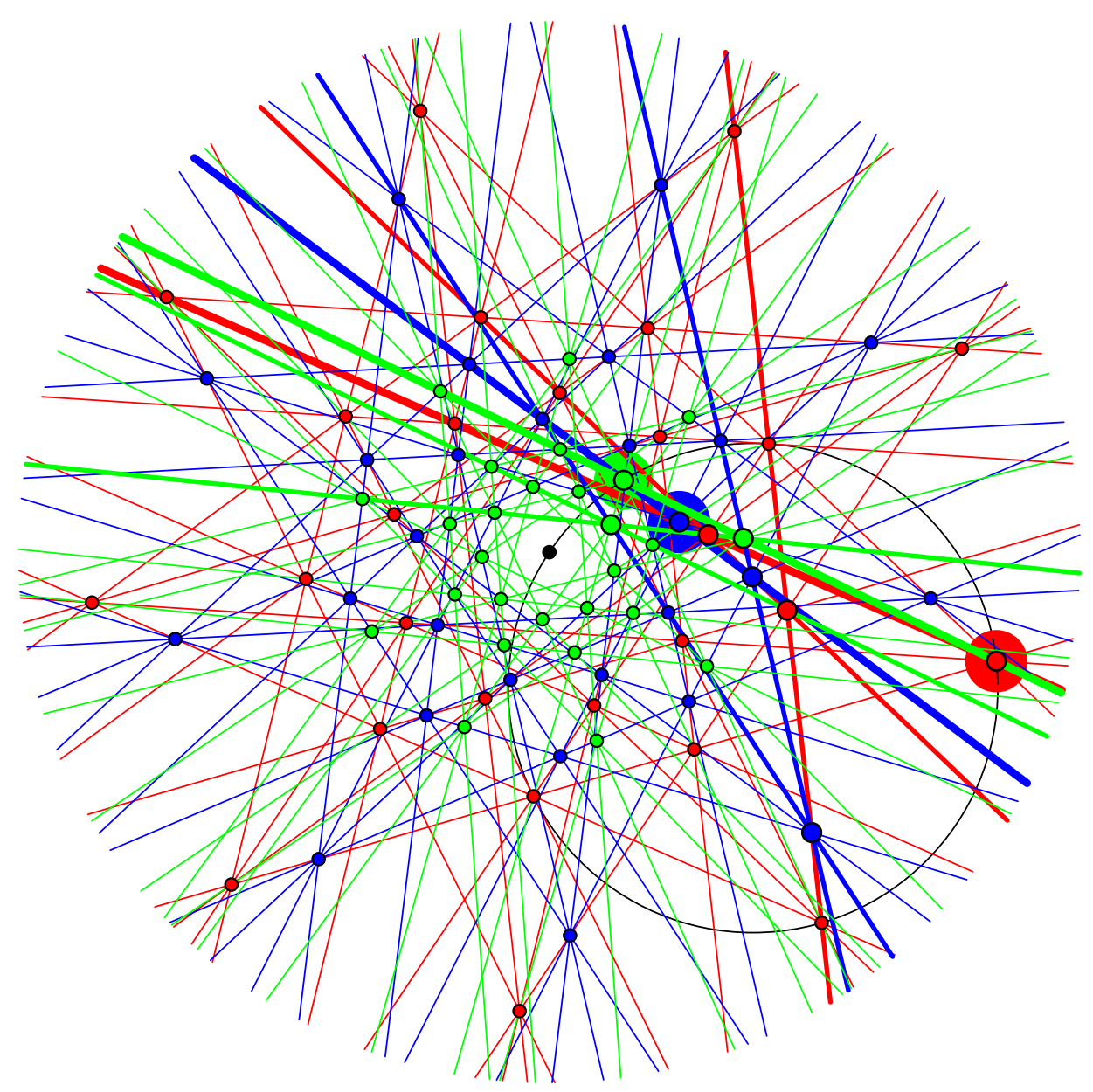

Figure 7: The smallest movable 5-configuration produced by Algorithm 2, an $\left(81_{5}\right)$ configuration, with initial celestial configuration $9 \#(4,3 ; 2,3 ; 1,3)$ shown in red, second celestial configuration $9 \#(2,3 ; 1,3 ; 4,3)$ shown in blue, and final celestial configuration $9 \#(1,3 ; 4,3 ; 2,3)$ shown in green. The point $\left(v_{1}^{1}\right)_{0}$ is highlighted in red, the line $\left(L_{1}^{1}\right)_{0}$ is the thickest red line, the point $\left(v_{1}^{2}\right)_{0}$ is highlighted in blue, and the line $\left(L_{1}^{2}\right)_{0}$ is the thickest blue line. The point $\left(v_{1}^{3}\right)_{0}$, which was constructed via the intersection of $\left(L_{1}^{2}\right)_{0}$ with the black circumcircle of span 1 through $\left(v_{1}^{1}\right)_{0}$, is highlighted in green, and $\left(L_{1}^{3}\right)_{0}$ is the thickest green line. Other 0th elements of symmetry classes are shown at medium weights. Already we have reached the limits of intelligibility of a small-scale diagram. 
If $k=2 j+1$ for some integer $j$, it is straightforward to show that

$$
2^{k}+1=2^{2 j+1}+1=(2+1) \sum_{i=0}^{2 j}(-1)^{i} 2^{i},
$$

so $2^{2 j}+1$ is clearly divisible by 3 , and $q=\sum_{i=0}^{2 j}(-1)^{i} 2^{i}$, which is odd.

Moreover, if $s_{i}=2^{i-1}$, then $\sum_{i=1}^{k} 2^{i-1}=2^{k}+1$. Thus, if $t_{i}=q$ for $i=1, \ldots, k$, then

$$
\sum_{i=1}^{k}\left(s_{i}-t_{i}\right)=\left(2^{k}+1\right)-(2 j+1) q
$$

is even, since both terms are odd.

It remains to show the cosine condition is fulfilled: that is, we need to show that for $q=$ $\frac{2^{k}+1}{3}$

$$
\prod_{i=1}^{k} \cos \left(\frac{2^{i-1} \pi}{3 q}\right)=\prod_{i=1}^{k} \cos \left(\frac{q \pi}{3 q}\right) .
$$

The right-hand side of equation (5.1) clearly evaluates to $\frac{1}{2^{k}}$. To see the left-hand side also evaluates to $\frac{1}{2^{k}}$, we use the following trigonometric identity, which can be proved using the identity $\sin (2 \theta)=2 \sin (\theta) \cos (\theta)$ and induction (see [10]):

$$
2^{k} \prod_{j=0}^{k-1} \cos \left(2^{j} a\right)=\frac{\sin \left(2^{j} a\right)}{\sin (a)}
$$

Applying this identity to the left-hand side of (5.1), we see that

$$
\begin{aligned}
\prod_{i=1}^{k} \cos \left(\frac{2^{i-1} \pi}{3 q}\right) & =\prod_{i=1}^{k} \cos \left(\frac{2^{i-1} \pi}{2^{k}+1}\right)=\frac{1}{2^{k}}\left(\frac{\sin \left(\frac{2^{k} \pi}{2^{k}+1}\right)}{\sin \left(\frac{\pi}{2^{k}+1}\right)}\right) \\
& =\frac{1}{2^{k}} \sin \left(\pi-\frac{\pi}{2^{k}+1}\right) \csc \left(\frac{\pi}{2^{k}+1}\right) \\
& =\frac{1}{2^{k}}\left(\sin (\pi) \cos \left(\frac{\pi}{2^{k}+1}\right)-\cos (\pi) \sin \left(\frac{\pi}{2^{k}+1}\right)\right) \csc \left(\frac{\pi}{2^{k}+1}\right) \\
& =\frac{1}{2^{k}}\left(0-(-1) \sin \left(\frac{\pi}{2^{k}+1}\right)\right) \csc \left(\frac{\pi}{2^{k}+1}\right) \\
& =\frac{1}{2^{k}},
\end{aligned}
$$

so the cosine condition is satisfied.

Theorem 5.4. There exists at least one 5-configuration with s continuous degrees of freedom, for infinitely many values of $s$. 
Proof. Use the cohort $3 q \#\left\{1,2, \ldots, 2^{k-1}\right\} ;\{\underbrace{q, q, \ldots, q}_{k}\}$ for $q=\frac{2^{k}+1}{3}, k$ odd and $k>$ 2 from Lemma 5.3; clearly, the sets $S$ and $T$ are disjoint. This produces a movable 5configuration with $k-2$ degrees of freedom for all odd $k \geq 3$.

\section{Open Questions}

Question 1. In [8], the Crossing Spans Lemma is generalized to allow larger and differently labelled subgraphs, as the Extended Crossing Spans Lemma. Are there interesting movable configurations that can be constructed from this generalization?

Question 2. This construction depends on two very simple geometric lemmas, which are straightforward to prove using basic Euclidean geometry. Are there other such useful lemmas? What techniques can be used, and which incidence theorems, to construct new configurations from known configurations while retaining useful symmetry properties?

Question 3. Finding movable 3-configurations is easy [6], and there are a number of known classes of movable 4-configurations [3, 4, 8, 14]. This paper presents a class of movable 5-configurations. Are there movable $k$-configurations for any $k>5$ ? For all $k>5$ ? In particular, are there movable 6-configurations?

\section{References}

[1] A. Berardinelli and L. W. Berman, Systematic celestial 4-configurations, Ars Math. Contemp. 7 (2014), 361-377.

[2] L. W. Berman, A characterization of astral $\left(n_{4}\right)$ configurations, Discrete Comput. Geom. 26 (2001), 603-612.

[3] L. W. Berman, Movable $\left(n_{4}\right)$ configurations, Electron. J. Combin. 13 (2006), Research Paper 104, 30, http://www.combinatorics.org/Volume_13/Abstracts/ v13i1r104.html.

[4] L. W. Berman, A new class of movable $\left(n_{4}\right)$ configurations, Ars Math. Contemp. 1 (2008), $44-50$.

[5] L. W. Berman, Constructing highly incident configurations, Discrete Comput. Geom. 46 (2011), 447-470, doi:10.1007/s00454-010-9279-7, http://dx.doi.org/10.1007/ s $00454-010-9279-7$.

[6] L. W. Berman, Geometric constructions for 3-configurations with non-trivial geometric symmetry, Electron. J. Combin. 20 (2013), Paper 9, 29.

[7] L. W. Berman and J. R. Faudree, Highly incident configurations with chiral symmetry, Discrete Comput. Geom. 49 (2013), 671-694, doi:10.1007/s00454-013-9494-0, http://dx. doi. org/10.1007/s00454-013-9494-0.

[8] L. W. Berman, J. R. Faudree and T. Pisanski, Polycyclic movable 4-configurations are plentiful, Discrete Comput. Geom. (Published Online 16 November 2015), doi:10.1007/ s00454-015-9749-z.

[9] L. W. Berman and L. Ng, Constructing 5-configurations with chiral symmetry, Electron. J. Combin. 17 (2010), Research Paper 2, 14, http://www.combinatorics.org/ Volume_17/Abstracts/v17i1r2.html. 
[10] W. A. Beyer, J. D. Louck and D. Zeilberger, Math Bite: A Generalization of a Curiosity that Feynman Remembered All His Life, Math. Mag. 69 (1996), 43-44, http: / /www • jstor. org/stable/2691393?origin=pubexport.

[11] M. Boben and T. Pisanski, Polycyclic configurations, European J. Combin. 24 (2003), 431-457, doi:10.1016/S0195-6698(03)00031-3, http://dx.doi.org/10 . $1016 / \mathrm{S} 0195-6698$ (03)00031-3.

[12] H. S. M. Coxeter, Self-dual configurations and regular graphs, Bull. Amer. Math. Soc. 56 (1950), 413-455.

[13] B. Grünbaum, Musings on an example of Danzer's, European J. Combin. 29 (2008), 1910 1918, doi:10.1016/j.ejc.2008.01.004, http://dx.doi.org/10.1016/j.ejc.2008. 01.004 .

[14] B. Grünbaum, Configurations of points and lines, volume 103 of Graduate Studies in Mathematics, American Mathematical Society, Providence, RI, 2009.

[15] B. Grünbaum and J. F. Rigby, The real configuration $\left(21_{4}\right)$, J. London Math. Soc. (2) 41 (1990), 336-346, doi:10.1112/jlms/s2-41.2.336, http://dx.doi.org/10.1112/ jlms/s2-41.2.336.

[16] D. Marušič and T. Pisanski, Weakly flag-transitive configurations and half-arc-transitive graphs, European J. Combin. 20 (1999), 559-570, doi:10.1006/eujc.1999.0302, http:// dx. doi. org/10.1006/eujc.1999.0302. 